\title{
Intrahepatic Cholestasis of Pregnancy: Natural History and Current Management
}

\author{
Rebecca Roediger, $\mathrm{MD}^{1}$ Jaquelyn Fleckenstein, $\mathrm{MD}^{1}$ \\ ${ }^{1}$ Division of Gastroenterology, Washington University School of \\ Medicine, St. Louis, Missouri \\ Semin Liver Dis 2021;41:103-108. \\ Address for correspondence Jaquelyn Fleckenstein, MD, 660 South \\ Euclid Avenue, Box 8124, Division of Gastroenterology, Washington \\ University School of Medicine, St. Louis, MO 63108 (e-mail: \\ jflecke@wustl.edu).
}

\begin{abstract}
Keywords

- cholestasis

- pregnancy

- pruritus

Intrahepatic cholestasis of pregnancy (ICP) is a common disorder in the second half of pregnancy characterized by pruritus and elevated serum bile acids (BAs) with spontaneous resolution after delivery. ICP carries a risk of adverse effects on the fetus which correlates with the degree of BA elevation. ICP occurs in genetically susceptible women as the reproductive hormones increase during pregnancy. Ursodeoxycholic acid is still considered the first-line treatment for ICP though it is of unproven benefit in preventing adverse effects on the fetus. Fetal complications, such as stillbirth, increase with gestational age, so preterm delivery is generally performed in cases of severe ICP, defined as BA levels above $40 \mu \mathrm{mol} / \mathrm{L}$. ICP may recur in future pregnancies and is associated with an increased risk for future hepatobiliary, immune mediated, and cardiovascular diseases. Children born of mothers with ICP have normal development but may have a risk for subsequent metabolic disease.
\end{abstract}

Intrahepatic cholestasis of pregnancy (ICP) is characterized by new-onset pruritus, typically presenting in the second half of pregnancy, associated with elevated bile acids (BAs) with no other underlying liver disease, and resolves after delivery. Maternal complications are minimal with the main risks coming from the fetus.

\section{Epidemiology}

ICP is the most common liver-related pregnancy disorder ${ }^{1}$ but is overall rare with a global prevalence ranging from 0.5 to $5.6 \%$ with geographic variation. ${ }^{2-4}$ The highest prevalence is in South America and Northern Europe. ${ }^{5,6}$ In Chile, the overall prevalence is $1.4 \%$ but can be as high as $15.6 \%$ in the Araucanos Indian population. ${ }^{3,7}$ In Sweden and Finland, ICP occurs in 1.5 and $1.3 \%$ of pregnancies, respectively. ${ }^{8,9}$ In the United States, the prevalence varies by demographics with a rate as low as $0.32 \%$ in Connecticut but as high as $5.6 \%$ in Latino populations in Los Angeles. ${ }^{3,10,11}$ There also appears to be a seasonal variation with ICP being more common in the winter months. ${ }^{5,6}$

published online

January 20, 2021

\section{Risk Factors}

There are several risk factors for ICP including hormonal, environmental, and genetic. ICP is more common in women who are pregnant with multiples. The incidence of ICP in twin pregnancies ranges from 14 to $22 \% 6,9$ and up to $43 \%$ in triplets. ${ }^{9}$ ICP is also more common in pregnancies that resulted from in vitro fertilization (IVF). The correlation with multiple pregnancies and IVF is likely related to the increased reproductive hormones in these women. Women with a previous history of liver-related diseases, specifically gallstones or hepatitis $C$, are at increased risk for ICP. ${ }^{4,5,9,12}$ Advanced maternal age and family history of ICP are also risk factors. $^{12}$ ICP is associated with other pregnancy-related disorders, carrying a three times increased risk of concomitant gestational diabetes and preeclampsia. ${ }^{13,14}$ Personal history of ICP is a significant risk for recurrence in subsequent pregnancies, ${ }^{12}$ with incidence of ICP as high as 60 to $70 \%$ in subsequent pregnancies. ${ }^{15}$ Rarely, pruritus and cholestasis can recur with the initiation of oral contraceptive pill (OCPs). ${ }^{12,15}$
(C) 2021. Thieme. All rights reserved. Thieme Medical Publishers, Inc., 333 Seventh Avenue, 18th Floor, New York, NY 10001, USA
DOI https://doi.org/ 10.1055/s-0040-1722264. ISSN 0272-8087. 


\section{Genetics}

The strong link of ICP in families along with variable incidence in certain racial groups suggests a genetic underpinning. Several genetic mutations have been described in ICP. The most common genetic mutation in ICP is of the adenosine triphosphate (ATP) binding cassette subfamily B member 4/multidrug resistance protein 3 (ABCB4/MDR3) gene. ${ }^{15}$ Mutations in this gene were first found in families with progressive familial intrahepatic cholestasis ${ }^{9}$ and now multiple genetic variations of this gene have been found in ICP. ${ }^{15,16}$ This gene encodes a transporter protein that moves phosphatidylcholine (PC) from the inner to the outer hepatocyte canalicular membrane. ${ }^{5} \mathrm{PC}$ is needed to prevent the detergent effects of BAs on the outer hepatocyte membranes since it forms mixed micelles. There is a clinical spectrum of diseases associated with $\mathrm{ABCB} 4$ deficiency, including ICP and gallbladder disease. ${ }^{17}$ Mutations in this gene may help explain the noted association of gallstone disease and ICP. ${ }^{8}$ Gamma-glutamyl transferase (GGT) is often elevated with mutations in this gene, though it is uncommonly elevated in ICP. $^{18}$

There are several other mutations in BA transporters that have been identified in ICP. ATP-binding cassette subfamily B member 11/bile salt export pump (ABCB11/BSEP) is the primary export pump for BA on the hepatocyte membrane. ${ }^{5}$ ATPase phospholipid transporting 8B1/familial intrahepatic cholestasis gene 1 (ATP8B1/FIC1) transports PC from the canaliculi into the hepatocyte. ${ }^{5,15}$ ATP-binding cassette subfamily $\mathrm{C}$ member 2 /multidrug resistance protein 2 (ABCC2/ MRP2) exports organic anions such as bilirubin glucuronide from the hepatocyte into bile. ${ }^{5}$ Nuclear receptor subfamily 1 group $\mathrm{H}$ member 4/farnesoid X receptor (NR1H4/FXR) is the main $B A$ receptor which regulates BA synthesis and transport. $^{5}$ The limited functioning of these mutated genes is hypothesized to be overwhelmed in the cholestatic state of increased reproductive hormones leading to ICP. ${ }^{18}$ Currently genetic testing is reserved for research studies, though testing for ABCB4 mutation can be considered if severe ICP presents early in gestation ${ }^{16,19}$ or if cholestasis persists after delivery. $^{18}$

\section{Presentation/Pathogenesis}

\section{Maternal}

ICP typically presents as new-onset pruritus in the second half of pregnancy which resolves within around 48 hours of delivery. ${ }^{2}$ In total, $80 \%$ of cases of ICP present after 30 weeks but ICP can present as early as 8 weeks. ${ }^{20}$ It tends to present earlier in multiple pregnancies. ${ }^{6}$ There is no evidence that earlier presentation correlates with more severe disease or worse fetal prognosis. ${ }^{2,21}$ Pruritus is typically of the hands and soles of the feet but can involve the entire body. It is worse at night and often interferes with the ability to sleep. ${ }^{6,20}$ Pruritus is not accompanied by a rash but there can be excoriations from scratching. ${ }^{6}$ There can be constitutional symptoms associated with cholestasis such as anorexia, malaise, and subclinical steatorrhea leading to fat-soluble vitamin deficiencies, ${ }^{6,12,22}$ but liver failure is not a sequela of ICP. ${ }^{15}$ Elevated international normalized ratio can be seen due to vitamin $\mathrm{K}$ deficiency. ${ }^{5}$ Elevated transaminases and bilirubin can occur but clinically evident jaundice is rare. ${ }^{6,18}$ Although pruritus typically resolves within days of delivery, BA and liver chemistries can take 2 to 8 weeks to normalize. ${ }^{5}$ If biochemistries remain elevated, other sources of liver disease should be investigated.

\section{Pathogenesis}

In ICP, genetic mutations affect the normal export, recycling, and synthesis of BA. BA transport to the gallbladder can be disrupted leading to more BA being excreted directly into the blood. ${ }^{6}$ The elevated levels of reproductive hormones estrogen and progesterone lead to cholestasis which can cause ICP in genetically susceptible individuals. ICP tends to occur in the second half of pregnancy when the reproductive hormones are the highest. It is more common in women with multiple pregnancies when reproductive hormones are at higher levels than singleton pregnancies. Finally, in some cases, hormone-induced cholestasis and pruritus recur with OCPs. Estrogen and progesterone desensitize the FXR pathway. ${ }^{20}$ Estrogen can also cause internalization of BA transport proteins resulting in cholestasis. ${ }^{3}$ Progesterone metabolites reduce export of BA by BSEP and competitively inhibit sodium taurocholate cotransporting polypeptide (NTCP), thus reducing hepatocyte reuptake of BA from the portal circulation. ${ }^{20}$

This disruption of normal BA physiology is speculated to lead to the pruritus and elevated serum BA in pregnant women. The BA levels do not correlate with severity of pruritus, though progesterone sulfate levels do, suggesting further interplay between reproductive hormones and BA in the pathophysiology of ICP. ${ }^{20}$

\section{Fetal}

ICP is correlated with multiple adverse perinatal outcomes, including preterm labor, fetal asphyxia, meconium staining of amniotic fluid, neonate respiratory distress, and intrauterine fetal death. ${ }^{3-5,9}$ A recent meta-analysis found that ICP had a higher risk of spontaneous preterm birth (odds ratio [OR]; 3.47), iatrogenic preterm birth (OR: 3.65), meconiumstained amniotic fluid (OR: 2.6 ), and need for the newborn to be admitted to the neonatal unit (OR: 2.12). ${ }^{13}$ Fetal outcomes are correlated with serum BA levels and there was not an increased risk of adverse outcomes for the fetus if the BA level was below $40 \mu \mathrm{mol} / \mathrm{L}^{2,18}$ The risk for preterm delivery and fetal asphyxia increases by 1 to $2 \%$ for each increase in $\mu \mathrm{mol} / \mathrm{L}$ of BA over $40 \mu \mathrm{mol} / \mathrm{L},{ }^{4}$ which is considered severe ICP. ${ }^{2,3,15}$ The risk of stillbirth is increased when BA levels are over $100 \mu \mathrm{mol} / \mathrm{L}^{3,13}$ However, ICP cases, in general, are mild and carry the same risk of fetal complications as healthy controls. $^{4}$

\section{Pathogenesis}

Fetal disease is thought to be due to increased BAs in the fetal circulation. In normal physiology, the fetus can start synthesizing BA by around 12 weeks and the placenta has a BA 
gradient that allows export of BA from the fetal circulation back into the maternal circulation. In ICP, this gradient is disrupted leading to increased levels of BA in the amniotic fluid, cord blood, and meconium. ${ }^{2,5,6,23}$ The main effects on the fetus in ICP are preterm labor, respiratory distress, meconium-stained amniotic fluid, fetal asphyxia, and intrauterine fetal death. ${ }^{3,13}$ BAs disrupt pulmonary surfactants leading to respiratory distress in neonates ${ }^{20}$ and increased fetal intestinal motility, potentially leading to meconiumstained amniotic fluid. ${ }^{3}$ BAs cause vasoconstriction of the placental chorionic veins and can induce fetal arrhythmias leading to fetal asphyxia and intrauterine fetal death. 3,24 Rodent studies have shown that BAs make the myometrium more sensitive to oxytocin and that there is a dose-dependent relationship of BA and myometrial contractility leading to spontaneous preterm birth. ${ }^{3}$

\section{Diagnostic Tests}

ICP is diagnosed in women in the second half of pregnancy who have pruritus, a total BA level $>10 \mu \mathrm{mol} / \mathrm{L}$ and no other causes for liver disease. ${ }^{3,15,18}$ Severe ICP is diagnosed with a BA above $40 \mu \mathrm{mol} / \mathrm{L}$. All women diagnosed with ICP should be screened for hepatitis $C$ if not previously tested and other causes of abnormal liver tests, and pruritus should be considered.

\section{Bile Acids}

Testing for elevated BAs is the most sensitive and specific test for ICP. ${ }^{5}$ Normal pregnancy levels of BA range from 0.3 to $9.8 \mu \mathrm{mol} / \mathrm{L}$ and do not increase as gestation progresses. ${ }^{3}$ Although BAs increase two to five times postprandially, peaking at around 90 minutes, ${ }^{25}$ random BA samples are generally obtained. ${ }^{25,26}$ A Cochrane review of BA found that elevated serum BAs have a 72 to $98 \%$ sensitivity and 81 to $97 \%$ specificity for ICP. ${ }^{26}$ Pruritus can precede elevation of BAs for up to 3 weeks, so it is recommended to continue to check BA levels in symptomatic women every 1 to 2 weeks after initial presentation even if BAs are initially normal. ${ }^{3}$

\section{Autotaxin}

Autotaxin is an enzyme which has myriad effects involving vascular and neuronal development in embryogenesis and is important for the formation of lysophosphatidic acid which has been found to be related to pruritus in rodent models. Autotaxin levels increase throughout pregnancy, tracking with gestational age. Autotaxin serum activity has been found to be higher in women with ICP compared with pregnant women with other forms of pruritus or liver disease. Using a threshold of $27 \mathrm{nmol} / \mathrm{mL} / \mathrm{min}$, autotaxin activity was found to have similar specificity and sensitivity to BA levels for the diagnosis of ICP. ${ }^{27}$ The autotaxin activity level does not fluctuate with food intake and so could be employed as a reliable diagnostic test for ICP in the future; however, more study is still needed.

\section{Liver Chemistries}

Liver chemistries are variably elevated in ICP. Transaminases can be elevated two to three times upper limit of normal, but can also remain normal in up to $30 \%$ of cases. ${ }^{27,28}$ The onset is variable and can occur before or after elevations in BAs are detected. ${ }^{3,15}$ Bilirubin is increased in approximately $10 \%$ of ICP cases and is usually a mild conjugated hyperbilirubinemia. ${ }^{6}$ Alkaline phosphatase is produced by the placenta, so it is elevated in normal pregnancies and is not a useful marker in diagnosis of ICP. GGT is usually normal or minimally elevated. $^{15}$

\section{Prothrombin Time}

Prothrombin time (PT) can be elevated due to poor absorption of vitamin $\mathrm{K}$, a fat soluble vitamin, in the setting of reduced BA recycling in $\mathrm{ICP}^{6}$

\section{Imaging}

In ICP, the liver parenchyma and biliary tree are normal, but the gallbladder volume is larger than in pregnant controls, though this is a nonspecific finding. ${ }^{3,5}$

\section{Liver Biopsy}

Liver biopsy is rarely indicated in the diagnostic work-up for ICP. However, the characteristic findings in ICP are canalicular cholestasis, bile plugs, and preserved portal tracts. ${ }^{3,15}$

\section{Pregnancy Management}

Ursodeoxycholic acid (UDCA) has been shown to improve pruritus and reduce both maternal and fetal BA levels in small randomized controlled trials (RCTs) and meta-analyses. ${ }^{3,20,29,30}$ UDCA is a hydrophilic BA which protects hepatocyte cell membranes from the toxic effects of hydrophobic BAs. ${ }^{29,31,32}$ It enhances expression of hepatocyte transporters BSEP, MRP4, and MRP3, thus stimulating biliary secretion, as well as preventing hepatocyte apoptosis and lowering levels of ethinyl-estradiol $17 \beta$-glucuronide, the main cholestatic metabolite of estrogen., 51,32 UDCA decreases the level of BA in fetal amniotic fluid and cord blood by restoring the physiologic placental BA gradient. ${ }^{23,24,33}$ Due to the relative rarity of adverse effects on the fetus and variability in early delivery, data confirming a decrease in fetal complications with the use of UDCA are lacking but meta-analyses have suggested an improvement in fetal outcomes. ${ }^{3,30}$

In the largest randomized placebo-controlled study of UDCA in ICP to date, there was no difference in fetal outcomes or decrease in maternal serum BA between UDCA and placebo groups but a small decrease in pruritus was found in the UDCA group. ${ }^{21} \mathrm{~A}$ total of 605 women were randomly assigned to placebo or UDCA. There was no difference in the primary outcome, which was a composite of perinatal death, preterm delivery, or neonatal intensive care unit admission after birth between the UDCA and placebo groups (adjusted risk ratio: 0.85 ; 95\% confidence interval: 0.62-1.15). A statistically significant improvement in maternal pruritus scores was found in the UDCA group but the difference, though statistically significant, was not clinically significant. The UDCA group also had more reduction in alanine aminotransferase but less reduction in BA compared with the 
placebo group. The incidence of the composite primary outcome was lower than expected, so the study could be underpowered to detect a difference. Previous studies have found that there is no increased risk of adverse prenatal outcomes when the BA level is below $40 \mu \mathrm{mol} / \mathrm{L},{ }^{2,18}$ and the risk of adverse fetal outcomes occurs most frequently when the BA level is above $100 \mu \mathrm{mol} / \mathrm{L}^{2,3,13}$ In this study only $24 \%$ of the participants had BA levels above $40 \mu \mathrm{mol} / \mathrm{L}$ and only $6.6 \%$ of participants had BA levels above $100 \mu \mathrm{mol} / \mathrm{L}$. The potential benefit of UDCA on fetal outcomes when BA levels are greater than $100 \mu \mathrm{mol} / \mathrm{L}$ remains unclear.

UDCA is still considered the first-line therapy for ICP although of unproven benefit to the fetus. It is safe in pregnancy and there have been no reported adverse effects to the mother or the fetus. ${ }^{18,21,30}$ The main reported side effect is mild diarrhea. ${ }^{34}$ UDCA is weight-based with the initial dosing of $15 \mathrm{mg} / \mathrm{kg} / \mathrm{day}$ given in two to three divided doses. ${ }^{3,18}$ UDCA is not specifically approved in breast feeding but is probably safe as breast milk itself contains significant amounts of UDCA at baseline. ${ }^{18}$ As pruritus typically resolves within days of delivery, UDCA is not usually necessary to continue after delivery.

\section{Dexamethasone}

Dexamethasone is not indicated as a treatment for ICP but is still recommended for fetal lung maturity in the event of preterm delivery. ${ }^{5,6,12,18}$

\section{Refractory Pruritus}

For pruritus refractory to standard UDCA dosing, the dose can be increased to $25 \mathrm{mg} / \mathrm{kg} /$ day or an additional agent can be added. ${ }^{18}$

\section{Rifampicin}

Rifampicin acts as a pregnane $\mathrm{X}$ receptor agonist. Its mechanism of action is not fully understood but it reduces bilirubin by increasing bilirubin conjugation and excretion. ${ }^{18,35}$ It works synergistically with UDCA to have an even greater effect on pruritus. An observational, retrospective study of UDCA and rifampicin found that when rifampicin was added to UDCA, BA decreased in over half of the women without reported adverse effects on the mother or fetus. ${ }^{35}$ Rifampicin does discolor urine, tears, and other bodily secretions, which is benign but patients should be counseled on this side effect prior to starting the medication. ${ }^{18}$

\section{Cholestyramine}

Cholestyramine, an anion exchange resin, has been considered to aid in the reduction of pruritus in ICP. However, while small studies have suggested an improvement in pruritus with cholestyramine, there is no effect of cholestyramine in reducing BA levels or improving liver chemistries. ${ }^{6}$ In a Cochrane review, cholestyramine was found to have less beneficial effect on pruritus and liver chemistries than UDCA and had more adverse effects. ${ }^{36}$ In addition, cholestyramine reduces intestinal absorption of BAs, including UDCA, and could worsen fat-soluble vitamin deficiencies in ICP. ${ }^{6,22}$ There is no evidence that cholestyramine provides benefit in the treatment of ICP. ${ }^{36}$

\section{S-Adenosyl-L-Methionine}

S-Adenosyl-L-methionine (SAMe) may improve cholestasis via several mechanisms. It is the main methyl-group donor in the synthesis of PC, which is necessary to form mixed micelles to protect hepatocyte membranes from BAs. ${ }^{5}$ SAMe also influences the fluidity of hepatocyte plasma membranes and stops the impairment of bile flow caused by ethinylestradiol by increasing the excretion of hormone metabolites. ${ }^{22,29}$ Despite its proposed ability to improve cholestasis, studies have provided mixed results on its efficacy. ${ }^{22,34,36,37}$ SAMe is given intravenously, usually 800 $\mathrm{mg} / \mathrm{day}$, and the most common adverse effect is thrombophlebitis from frequent intravenous infusions. ${ }^{6,22}$ SAMe is generally reserved for the treatment of pruritus refractory to other additional agents.

\section{Symptomatic Relief}

Antihistamines can reduce pruritus and cause drowsiness, which can aid in treating the pruritus-associated insomnia in ICP. ${ }^{6}$ However, antihistamines have no effect on reducing serum BA, liver function tests, or adverse effects on the fetus. Topical emollients, such as aqueous creams with $2 \%$ menthol, can provide relief from pruritus but do not have any systemic effects. ${ }^{6}$

\section{Coagulopathy}

There is concern for coagulopathy in ICP related to vitamin K deficiency caused by reduced absorption of fat-soluble vitamins from impaired BA enterohepatic circulation in ICP. However, in a prospective, observational study, no difference was found in blood loss from vaginal deliveries between women with ICP and healthy controls. ${ }^{4}$ In the recent large RCT of UDCA, compared with placebo for treatment of ICP, though both the placebo and UDCA groups had vaginal blood loss within the normal range, the UDCA had less blood loss compared with placebo. ${ }^{21}$

\section{Vitamin $\mathrm{K}$}

Both liver and obstetric society guidelines recommend supplementing vitamin $\mathrm{K}$ in the setting of elevated PT and some guidelines recommend supplementing vitamin $\mathrm{K}$ prior to delivery in all cases of ICP. ${ }^{12}$

\section{Early Delivery}

Since ICP carries a risk of increased stillbirth, particularly as gestational age increases, many guidelines recommend early delivery. ${ }^{12}$ In a retrospective study of women with ICP, there was a $7 \%$ incidence of stillbirth in singleton pregnancies at a mean gestational age of 38 weeks and a $22 \%$ incidence of stillbirth in multiple pregnancies at a mean gestational age of 37 weeks. ${ }^{38}$ These stillbirth rates are likely higher than as seen in the normal population as women who developed complications were more likely to respond to recruitment for this retrospective study. Other studies of stillbirth in ICP have estimated the risk to be relatively rare, around $1.74 \%$ of pregnancies. ${ }^{12}$ Risk for stillbirth is more elevated in ICP where BA levels are greater than $100 \mu \mathrm{mol} / \mathrm{L}^{3}$ so estimates of incidence of intrauterine fetal death are likely skewed by 
the number of women with this severe form of ICP included in each study. Regardless of the true incidence of stillbirth which varies in studies, it does appear that intrauterine fetal death increases with gestational age, so induction is often performed around 37 weeks to reduce the risk of stillbirth while minimizing risk of prematurity. ${ }^{3}$ In cases where BA levels are above $100 \mu \mathrm{mol} / \mathrm{L}$, there is fetal distress, or maternal complications, and delivery earlier than 37 weeks is considered on a case-by-case basis to balance the risks of intrauterine fetal death with the risks of prematurity. ${ }^{12}$ Regular monitoring of BA is generally performed after diagnosis but specific guidelines vary with respect to timing and frequency of testing. $^{12}$

\section{Fetal Management}

Many obstetricians increase fetal monitoring via cardiotocography or fetal activity monitoring in women with ICP. However, fetal monitoring has not been shown to predict or reduce the risk of intrauterine fetal death. Currently, no specific fetal monitoring is recommended. ${ }^{12,18}$

\section{Long-Term Prognosis}

\section{Maternal Prognosis}

Immediately post-delivery, maternal prognosis is excellent and there are no contraindications to breastfeeding for women who had ICP. ${ }^{12}$ Pruritus typically resolves in 48 hours and liver biochemical abnormalities normalize by 4 weeks. ${ }^{6}$ ICP recurs in subsequent pregnancies for 60 to $70 \%$ of women, though it recurs less frequently after ICP in pregnancy with multiples. ${ }^{5,15}$ ICP can recur with the use of hormonal OCPs and women with a history of ICP should be counseled of this risk prior to initiating OCPs. The risk of recurrence is not a contraindication to low-dose dual hormonal OCPs, progesterone OCPs, or intrauterine devices. $^{12,15}$

There is an increased incidence of subsequent hepatobiliary disease in women with previous ICP., ${ }^{3,6,8,15}$ A Swedish study that used national registry data to compare long-term outcomes for women who had ICP with age-matched controls giving birth in the same year found that women with ICP were 2.47 times more likely to develop hepatobiliary disease than controls, specifically chronic hepatitis (hazard ratio [HR]: 5.96), liver fibrosis or cirrhosis (HR: 5.11), hepatitis C (HR: 4.16), cholangitis (HR: 4.22), and gallstone disease (HR: 2.72). ${ }^{8}$ Women who had ICP also have an increased risk for liver-related cancers (HR: 3.61) even after adjusting for increased prevalence of hepatitis $C$ in this population. An increased risk of subsequent immune-mediated diseases (HR: 1.9), specifically thyroid disease, diabetes, Crohn's disease, psoriasis, inflammatory polyarthropathy, and for cardiovascular disease (HR: 1.12 ) has also been found. ${ }^{14}$

\section{Fetal Prognosis}

Newborns that survive the perinatal period do well and have normal development. There may be an increased risk for metabolic disease in children born from women with ICP. ${ }^{14}$

\section{Conclusion}

ICP presents as new-onset pruritus of the hands and soles of the feet but can involve any part of the body with onset typically in the second half of pregnancy and spontaneously resolves after delivery. Elevated serum BA levels are the most sensitive and specific test for ICP and adverse effects on the fetus correlate with the BA level. The risk of adverse effects on the fetus such as preterm birth and meconium staining of amniotic fluid increases as BA levels elevate above $40 \mu \mathrm{mol} / \mathrm{L}$ and stillbirth risk increases at BA levels above $100 \mu \mathrm{mol} / \mathrm{L}$. UDCA is the main therapeutic option with previous studies demonstrating effects on reducing pruritus and serum BA levels, though a recent large RCT was not able to replicate these findings. Vitamin K should be supplemented if the PT is prolonged. The decision for a planned preterm birth is individualized. Women with ICP are at a higher risk for recurrent ICP, subsequent diagnosis of hepatobiliary disease, hepatocellular carcinoma, immune-mediated diseases, and cardiovascular disease. All women with ICP should be screened for hepatitis $C$ if not previously tested. Children born of women with ICP may have a higher risk for metabolic disease later in life, but otherwise have normal development.

\section{Main Concepts and Learning Points}

- Intrahepatic cholestasis of pregnancy (ICP) is characterized by new-onset pruritus typically in the second half of pregnancy which resolves after delivery. Adverse effects on the fetus such as preterm birth, fetal asphyxia, meconium staining of amniotic fluid, and intrauterine fetal death are rare sequelae of severe ICP.

- Bile acids (BAs) are the most sensitive and specific diagnostic test for ICP. BA level greater than $10 \mu \mathrm{mol} / \mathrm{L}$ with pruritus and no concomitant liver disease is diagnostic of ICP. Severe ICP is defined as BA levels greater than $40 \mu-$ $\mathrm{mol} / \mathrm{L}$. The risk of stillbirth increases with BA levels above $100 \mu \mathrm{mol} / \mathrm{L}$.

- Ursodeoxycholic acid is the current first-line treatment, though it has unclear benefit on adverse effects on the fetus.

- Women with ICP are at higher risk for subsequent diagnosis of recurrent ICP, future diagnosis of hepatobiliary disease, hepatobiliary cancer, immune-mediated diseases, and cardiovascular disease. Children born of mothers with ICP may have a higher risk of metabolic diseases later in life, but otherwise have normal development.

Conflict of Interest

None declared.

\section{References}

1 Allen AM, Kim WR, Larson JJ, et al. The epidemiology of liver diseases unique to pregnancy in a US community: a populationbased study. Clin Gastroenterol Hepatol 2016;14(02):287.e1, 2-294.e1, 2

2 Di Mascio D, Quist-Nelson J, Riegel M, et al. Perinatal death by bile acid levels in intrahepatic cholestasis of pregnancy: a systematic 
review. J Matern Fetal Neonatal Med 2019 (e-pub ahead of print). . Doi: 10.1080/14767058.2019.1685965

3 Wood AM, Livingston EG, Hughes BL, Kuller JA. Intrahepatic cholestasis of pregnancy: a review of diagnosis and management. Obstet Gynecol Surv 2018;73(02):103-109

4 Glantz A, Marschall HU, Mattsson LÅ Intrahepatic cholestasis of pregnancy: Relationships between bile acid levels and fetal complication rates. Hepatology 2004;40(02):467-474

5 Geenes V, Williamson C. Intrahepatic cholestasis of pregnancy. World J Gastroenterol 2009;15(17):2049-2066

6 Williamson C, Geenes V. Intrahepatic cholestasis of pregnancy. Obstet Gynecol 2014;124(01):120-133

7 Reyes H, Gonzalez MC, Ribalta J, et al. Prevalence of intrahepatic cholestasis of pregnancy in Chile. Ann Intern Med 1978;88(04): 487-493

8 Marschall HU, Wikström Shemer E, Ludvigsson JF, Stephansson O. Intrahepatic cholestasis of pregnancy and associated hepatobiliary disease: a population-based cohort study. Hepatology 2013; 58(04):1385-1391

9 Savander M, Ropponen A, Avela K, et al. Genetic evidence of heterogeneity in intrahepatic cholestasis of pregnancy. Gut 2003; 52(07):1025-1029

10 Lee RH, Goodwin TM, Greenspoon J, Incerpi M. The prevalence of intrahepatic cholestasis of pregnancy in a primarily Latina Los Angeles population. J Perinatol 2006;26(09):527-532

11 Laifer SA, Stiller RJ, Siddiqui DS, Dunston-Boone G, Whetham JCG. Ursodeoxycholic acid for the treatment of intrahepatic cholestasis of pregnancy. J Matern Fetal Med 2001;10(02):131-135

12 Bicocca MJ, Sperling JD, Chauhan SP. Intrahepatic cholestasis of pregnancy: Review of six national and regional guidelines. Eur J Obstet Gynecol Reprod Biol 2018;231(231):180-187

13 Ovadia C, Seed PT, Sklavounos A, et al. Association of adverse perinatal outcomes of intrahepatic cholestasis of pregnancy with biochemical markers: results of aggregate and individual patient data meta-analyses. Lancet 2019;393(10174):899-909

14 Wikström Shemer EA, Stephansson O, Thuresson M, Thorsell M, Ludvigsson JF, Marschall HU. Intrahepatic cholestasis of pregnancy and cancer, immune-mediated and cardiovascular diseases: a population-based cohort study. J Hepatol 2015;63(02):456-461

15 Bacq Y, Sentilhes L. Intrahepatic cholestasis of pregnancy: diagnosis and management. Clin Liver Dis (Hoboken) 2014;4(03):58-61

16 Reichert MC, Lammert F. ABCB4 gene aberrations in human liver disease: an evolving spectrum. Semin Liver Dis 2018;38(04): 299-307

17 Sticova E, Jirsa M. ABCB4 disease: many faces of one gene deficiency. Ann Hepatol 2020;19(02):126-133

18 European Association for the Study of the Liver. EASL clinical practice guidelines: management of cholestatic liver diseases. J Hepatol 2009;51(02):237-267

19 Yeap SP, Harley H, Thompson R, et al. Biliary transporter gene mutations in severe intrahepatic cholestasis of pregnancy: diagnostic and management implications. J Gastroenterol Hepatol 2019;34(02):425-435

20 Dixon PH, Williamson C. The pathophysiology of intrahepatic cholestasis of pregnancy. Clin Res Hepatol Gastroenterol 2016;40 (02):141-153

21 Chappell LC, Bell JL, Smith A, et al;PITCHES study group. Ursodeoxycholic acid versus placebo in women with intrahepatic cholestasis of pregnancy (PITCHES): a randomised controlled trial. Lancet 2019;394(10201):849-860
22 Ribalta J, Reyes H, Gonzalez MC, et al. S-adenosyl-L-methionine in the treatment of patients with intrahepatic cholestasis of pregnancy: a randomized, double-blind, placebo-controlled study with negative results. Hepatology 1991;13(06):1084-1089

23 Brites D. Intrahepatic cholestasis of pregnancy: changes in maternal-fetal bile acid balance and improvement by ursodeoxycholic acid. Ann Hepatol 2002;1(01):20-28

24 Lofthouse EM, Torrens C, Manousopoulou A, et al. Ursodeoxycholic acid inhibits uptake and vasoconstrictor effects of taurocholate in human placenta. FASEB J 2019;33(07):8211-8220

25 Manna LB, Ovadia C, Lövgren-Sandblom A, et al. Enzymatic quantification of total serum bile acids as a monitoring strategy for women with intrahepatic cholestasis of pregnancy receiving ursodeoxycholic acid treatment: a cohort study. BJOG 2019;126 (13):1633-1640

26 Manzotti C, Casazza G, Stimac T, Nikolova D, Gluud C. Total serum bile acids or serum bile acid profile, or both, for the diagnosis of intrahepatic cholestasis of pregnancy. Cochrane Database Syst Rev 2019;7(07):CD012546

27 Kremer AE, Bolier R, Dixon PH, et al. Autotaxin activity has a high accuracy to diagnose intrahepatic cholestasis of pregnancy. J Hepatol 2015;62(04):897-904

28 Lammert F, Marschall H-U, Glantz A, Matern S. Intrahepatic cholestasis of pregnancy: molecular pathogenesis, diagnosis and management. J Hepatol 2000;33(06):1012-1021

29 Zhang Y, Lu L, Victor DW, Xin Y, Xuan S. Ursodeoxycholic acid and S-adenosylmethionine for the treatment of intrahepatic cholestasis of pregnancy: a meta-analysis. Hepat Mon 2016;16(08): e38558

30 Bacq Y, Sentilhes L, Reyes HB, et al. Efficacy of ursodeoxycholic acid in treating intrahepatic cholestasis of pregnancy: a metaanalysis. Gastroenterology 2012;143(06):1492-1501

31 Marschall HU, Wagner M, Zollner G, et al. Complementary stimulation of hepatobiliary transport and detoxification systems by rifampicin and ursodeoxycholic acid in humans. Gastroenterology 2005;129(02):476-485

32 Beuers U. Drug insight: mechanisms and sites of action of ursodeoxycholic acid in cholestasis. Nat Clin Pract Gastroenterol Hepatol 2006;3(06):318-328

33 Estiú MC, Monte MJ, Rivas L, et al. Effect of ursodeoxycholic acid treatment on the altered progesterone and bile acid homeostasis in the mother-placenta-foetus trio during cholestasis of pregnancy. Br J Clin Pharmacol 2015;79(02):316-329

34 Zhang L, Liu XH, Qi HB, et al. Ursodeoxycholic acid and Sadenosylmethionine in the treatment of intrahepatic cholestasis of pregnancy: a multi-centered randomized controlled trial. Eur Rev Med Pharmacol Sci 2015;19(19):3770-3776

35 Geenes V, Chambers J, Khurana R, et al. Rifampicin in the treatment of severe intrahepatic cholestasis of pregnancy. Eur J Obstet Gynecol Reprod Biol 2015;189:59-63

36 Gurung V, Middleton P, Milan SJ, Hague W, Thornton JG. Interventions for treating cholestasis in pregnancy. Cochrane Database Syst Rev 2013;2013(06):CD000493

37 Azzaroli F, Turco L, Lisotti A, Calvanese C, Mazzella G. The pharmacological management of intrahepatic cholestasis of pregnancy. Curr Clin Pharmacol 2011;6(01):12-17

38 Williamson C, Hems LM, Goulis DG, et al. Clinical outcome in a series of cases of obstetric cholestasis identified via a patient support group. BJOG 2004;111(07):676-681 